Cahiers de recherches médiévales

La Noblesse en question (XIII $-\mathrm{XV}^{\mathrm{e}} \mathrm{s}$.)

\title{
Un coup de foudre
}

La recherche anglo-saxonne sur le Roman de Silence

\section{F. Regina Psaki}

\section{(2) OpenEdition}

Journals

Édition électronique

URL : https://journals.openedition.org/crm/776

DOI : $10.4000 / \mathrm{crm} .776$

ISSN : 1955-2424

Éditeur

Honoré Champion

Édition imprimée

Date de publication : 30 décembre 2006

Pagination : 287-303

ISSN : 1272-9752

Référence électronique

F. Regina Psaki, « Un coup de foudre », Cahiers de recherches médiévales [En ligne], 13 | 2006, mis en ligne le 27 novembre 2009, consulté le 15 décembre 2022. URL : http://journals.openedition.org/crm/ 776 ; DOl : https://doi.org/10.4000/crm.776 


\title{
rM
}

\section{Un coup de foudre : la recherche anglo-saxonne sur le Roman de Silence*}

\author{
«A pluri-vocal text may be perspectivally organized and scat- \\ ter in its semiotic landscape signposts of reading, markers to \\ indicate, not precise meanings to be extracted, but the kinds of \\ questions to be raised». \\ Peter Haidu, The Subject Medieval / Modern ${ }^{1}$
}

Cet article est dédié au professeur Joan Ferrante, ambassadrice presciente et énergique du Roman de Silence

Bien qu'écrit en ancien français au XIII ${ }^{\mathrm{e}}$ siècle et d'après les paramètres du roman courtois continental, le Roman de Silence ne survécut qu'en GrandeBretagne, dans un manuscrit unique, oublié pendant plusieurs siècles dans le grenier d'un manoir anglais, le Wollaton Hall, à Nottingham². C'est encore en Angleterre qu'avec le manuscrit, le roman fut redécouvert; un chercheur britannique fut le premier à l'éditer et à le publier plusieurs décennies plus tard ${ }^{3}$; deux traductions en langue anglaise lui permettent d'être utilisé aujourd'hui dans l'enseignement de nombreuses universités nord-américaines ${ }^{4}$; c'est enfin toujours en anglais - du moins jusqu'à présent - qu'il a connu son plus grand nombre d'admirateurs et d'exégètes. Le Roman de Silence n'a pas immédiatement suscité l'intérêt des médiévistes francophones et n'a pas encore été repris ni mentionné dans les histoires de la littérature, dans les dictionnaires ou encyclopédies du Moyen Âge, ou dans les séries

\footnotetext{
${ }^{*}$ Note de la rédaction : cet article de F. Regina Psaki vient compléter le petit dossier consacré au Roman de Silence publié dans le dernier numéro des Cahiers de Recherches Médiévales, 12, 2005, p. 205-245. Article traduit de l'anglais par M. Vanscheeuwijck, University of Oregon.

${ }^{1}$ Peter Haidu, «Problematizing Identity : Silence», dans son livre The Subject Medieval / Modern: Text and Governance in the Middle Ages, Stanford: Stanford University Press, 2004, p. 239-265. La citation se trouve p. 240.

${ }^{2}$ Sur Mi LM 6, voir Frederick A. G. Cowper, "Origins and Peregrinations of the LavalMiddleton Manuscript », Nottingham Medieval Studies 3 (1959), p. 3-18.

${ }^{3}$ Le roman a grand besoin d'une nouvelle édition. L'édition de Lewis Thorpe (publiée en série et en livre) fut corrigée par Sarah Roche-Mahdi (voir note 4), suivant les corrections de Félix Lecoy, mais une toute nouvelle édition approfondie offrira aux chercheurs un meilleur point de départ fort attendu. Voir Heldris de Cornuälle, Le Roman de Silence, éd. Lewis Thorpe, Cambridge: Heffer, 1972 et Félix Lecoy, "Corrections : Le Roman de Silence d'Heldris de Cornuälle », Romania 99 (1978), p. 109-125.

${ }^{4}$ Regina Psaki, trad. Le Roman de Silence, New York : Garland (Garland Library of Medieval Literature 63B), 1991; et Sarah Roche-Mahdi, éd. et trad. Silence : A Thirteenth-Century French Romance, East Lansing, MI : Colleagues Press, 1992.
} 
d'éditions les plus importantes de textes en ancien français. Heureusement, un nouvel ensemble d'études excellentes, une traduction récente, ainsi qu'une nouvelle édition à paraître (j'en suis ravie) du roman, ont démontré qu'il a enfin trouvé un public en français ${ }^{5}$.

Ce n'est bien sûr pas à une médiéviste américaine de spéculer sur la question de savoir pourquoi le roman a mis plus de temps à émerger en France et dans les études médiévales francophones que dans la recherche anglo-américaine. La distance n'offre pas forcément l'avantage de la clarté et parfois elle peut même créer de grossières distorsions. J'ai l'intention, dans cet essai, d'explorer la réception du roman dans les études médiévales anglophones, sans pour cela proposer un parcours strictement chronologique. Je n'offrirai pas non plus un profil approfondi ni une bibliographie annotée, étant donné qu'un simple article ne peut se prêter à une description détaillée de l'approche, de la thèse, des arguments, des découvertes ou des omissions de chacun des nombreux articles et chapitres désormais dédiés au Roman de Silence $e^{6}$. Par contre, je tiens à présenter un survol général des contributions et des débats actuels à propos de ce texte extraordinaire d'Heldris de Cornuälle ${ }^{7}$.

Lorsque le Roman de Silence fut remarqué dans les études médiévales anglophones dans les années 1980, ce fut un véritable coup de foudre. Le texte est symptomatique de la façon dont certaines productions culturelles du passé- quoique connues, ou dont on connaissait au moins l'existence - ont pu demeurer invisibles, jusqu'à ce qu'un nombre donné de questions convergent ensuite pour les rapporter au cœur de l'attention. Les quêtes, qui ont promu le roman au centre de notre intérêt furent, en premier lieu, celles déterminées par les «women's studies » et la théorie post-structuraliste, et ensuite, la recherche médiévale féministe et les «gender studies $»^{8}$. La consolidation et même la canonisation de ces «quêtes» dans la recherche

${ }^{5}$ L'édition à paraître en question est celle que D. James-Raoul est en train d'achever pour les éditions Honoré Champion.

${ }^{6}$ Sous les auspices du Feminist Humanities Project du Center for the Study of Women in Society de la University of Oregon, je suis en train de développer un outil de recherche électronique, qui permet de telles descriptions systématiques que l'on peut mettre à jour, bien que pour des raisons de droits d'auteurs, le site ne soit pas encore public (http://server.fhp.uoregon.edu/dtu/sites/silence/index.html-ssi).

${ }^{7}$ En outre j'offre un supplément à l'excellente bibliographie du professeur Silvère Menegaldo fournie dans les Cahiers de Recherches Médiévales 12 (2005), p. 207-210.

${ }^{8}$ Les termes gender et gender studies demandent ici une petite explication. En anglais, le mot français genre dénote des formes et des catégories littéraires, tandis que le mot anglais gender réfère tant au genre grammatical qu'à l'identité qui se rapporte, sans nécessairement y correspondre, au sexe biologique. Dans mon article, j'utilise le mot anglais gender pour indiquer l'identité située au long du spectre défini par le «masculin» et le «féminin » (plutôt que les catégories «homme » et «femme») et je réserve le mot français genre pour les types littéraires. Par contre, j'emploie l'expression gender studies pour indiquer le vaste champ de recherche dans les dimensions culturelles, comportementales, sociales et autres, d'identité de genre et de différence de genre. Les gender studies, à l'instar des women's studies et des études féministes, n'explorent pas seulement les expériences des femmes et l'idéologie de la différence entre les sexes, mais aussi les intersections complexes d'identité et de différence entre les sexes, l'identité sexuelle et les rôles, les conventions et les comportements associés au gender. La masculinité (ou plutôt les masculinités), les féminités et les autres identités de sexe sont traitées de façon équivalente dans le domaine des gender studies, discipline qui a ten- 
sur le roman eurent l'effet de générer une attention nouvelle aux modes dans lesquels le texte était fondé dans son cadre historique et matériel et reflété dans celuici. Enfin, le Roman de Silence fut également adapté en vue d'»exécutions » par des médias variés, un processus qui met au jour d'autres dimensions du roman que l'analyse strictement littéraire ou historique. Ce compte rendu très sommaire servira à établir la discussion qui suit, tout en traçant les grands traits des sujets du roman, mis en évidence par la critique anglophone des années 1980 jusqu'aujourd'hui'.

\section{Théorie post-structuraliste et «women's studies »}

Lorsque le Roman de Silence fut publié en livre en 1972, il apparut au milieu d'une révolution intellectuelle en cours et dans laquelle beaucoup de catégories étaient en train d'être interrogées et déstabilisées. L'essor du féminisme et du poststructuralisme fut rapidement reflété dans les écrits sur le roman et les deux points de vue furent entrelacés dans les premières études fondamentales de Kate Cooper (1985), de Howard Bloch (1983 et 1986) et de Peter Allen $(1989)^{10}$. Les trois critiques accentuèrent la façon dont le texte interroge «the workings of poetic creation, [...] the sexuation of language, [and] the possibilities of pure signification », provoquant ainsi un refus radical de détermination dans les trois dimensions ${ }^{11}$. Pour Bloch, «Silence represents the systematic refusal of univocal meaning ${ }^{12}$ ». Allen, quant à lui, se concentre sur la façon dont le langage du texte et le cadre de son manuscrit fonctionnent pour préserver une ambiguité programmatique; l'inaccessibilité du sens déterminé de ce texte distant reflèterait ainsi l'abîme entre les modes de lecture précartésiens et modernes.

Dans sa récente analyse élaborée du Roman de Silence et de L'Enfant de sable de Tahar Ben Jelloun, Erika Hess a comparé l'épistémologie, l'esthétique et la technique narrative médiévale et moderne. Elle examine la façon dont les deux textes se rencontrent dans leur «construction of gender, $[\ldots]$ narrative openendedness

dance à se considérer comme étant «au-delà » des women's studies et des études féministes, au sein desquelles elles se sont développées.

${ }^{9}$ Cette synthèse simplifie peut-être trop l'histoire de la critique, dans le sens où elle sépare des ensembles de sujets qui se superposent dans la plupart des analyses. Les études mentionnées ne se situent pas proprement dans des camps séparés, et j'en parlerai à plusieurs reprises.

${ }^{10}$ Kate Mason Cooper, "Elle and $L$ : Sexualized Textuality in Le Roman de Silence », Romance Notes 25.3 (1985), p. 341-360; R. Howard Bloch, «Silence and Holes: The Roman de Silence and the Art of the Trouvère », Yale French Studies 70 (1986), p. 81-99; Peter L. Allen, «The Ambiguity of Silence : Gender, Writing, and Le Roman de Silence », dans Sign, Sentence, Discourse : Language in Medieval Thought and Literature, éd. Julian N. Wasserman et Lois Roney, Syracuse : Syracuse UP, 1989, p. 98-112. Cette trajectoire de recherche persiste encore plus récemment chez quelques critiques du Roman de Silence, dont Suzanne Kocher, «Undermining Oppositionality: The Romance of Silence's Nature / Nurture Debate Complicated by the Rhymes of apelier (to name) and celer (to conceal) », Romance Languages Annual 7 (1995), p. 95-99; Gloria Gilmore, «Le Roman de Silence: Allegory in Ruin or Womb of Irony? » Arthuriana 7.2 (1997), p. 111-123; et Erin Labbie, «The Specular Image of the Gender-Neutral name: Naming Silence in Le Roman de Silence », Arthuriana 7.2 (1997), p. 63-77.

${ }^{11}$ Cooper, art. cit., p. 343.

${ }^{12}$ Bloch, art. cit., p. 88. 
and polysemy, [and] questioning of the primacy of the sexed body in gender identity » en vue de thématiser et de privilégier le sens non-déterminé, plutôt que la signification univoque ${ }^{13}$. Cette exégèse complexe du roman et de son double postmoderne fait fructifier les thèmes et les intérêts de Cooper, de Bloch et d'Allen avec un degré de spécificité et de nuance impossible à reproduire dans cet article. Déjà le titre des chapitres offre une idée claire de l'ampleur et de la profondeur de cette étude exceptionnellement détaillée : «narrative apertures and physical hybridism: the questioning of authoritative meaning »; "passing for true: gender as performance»; «parallel ambiguities : narrative and generic»; «the ultimate challenge to the primacy of the sexed body ».

Tandis que tous ces critiques furent certes attentifs au rôle unique du gender dans le roman, le texte examine, de ce point de vue, plutôt de textualité que de la sexualité et des sexes ou des genders, la nature et la «culture (noreture), les hommes et les femmes. Suivirent d'autres études, qui ont pris la politique sexuelle du roman plus comme un point d'intérêt en soi que comme une «metaphor for poetic practice and linguistic difference $\gg^{14}$. Ma première rencontre, encore étudiante, avec le Roman de Silence, eut lieu en 1985, lors d'une conférence à la Cornell University du professeur Joan Ferrante de la Columbia University ${ }^{15}$. Dans son allocution, Ferrante présenta le texte comme une preuve fascinante du fait qu'au Moyen Âge, les gens reconnaissaient vivement le couple nature / noreture comme une influence sur le caractère et le destin de l'individu ${ }^{16}$, particulièrement dans le contexte de l'identité sexuelle et du gender. À ses débuts, la critique féministe s'est intéressée surtout et exprès au «point d'accroche» de la trame de l'histoire, c'est-à-dire à l'idée qu'une fille éduquée comme un garçon puisse non seulement égaler, mais surpasser tous les chevaliers masculins ${ }^{17}$. La précondition de cette trame était la possibilité de maintenir, ne fût-ce que de façon spéculative et momentanée, l'hypothèse que ce qui sépare fondamentalement les hommes des femmes est leur éducation et leur assimilation culturelle et non pas leur essence ou potentiel innés. Bien sûr, les modernes se sentirent entièrement capables de considérer une telle hypothèse, mais ils exprimèrent toutefois un plaisir émerveillé dans le fait qu'un poète médiéval ait pu présenter ceci

${ }^{13}$ Erika E. Hess, Literary Hybrids : Cross-Dressing, Shapeshifting, and Indeterminacy in Medieval and Modern French Narrative (New York / London : Routledge, 2004), p. 3.

${ }^{14}$ Jane Burns, Courtly Love Undressed: Reading Through Clothes in Medieval Literature (Philadelphia : University of Pennsylvania Press, 2002), p. 69.

${ }^{15}$ Joan Ferrante, «Public Postures and Private Maneuvers : Roles Medieval Women Play », dans Women and Power in the Middle Ages, éd. Mary Erler et Maryanne Kowaleski (Athens, GA : University of Georgia Press, 1988), p. 213-229. Au début, les activités du prof. Ferrante, dont le séminaire NEH qu'elle dirigea en 1981, ont donné beaucoup de presse au Roman de Silence.

${ }^{16}$ La culture populaire et générale tend à ne pas attribuer au Moyen Âge la connaissance de ce couple d'opposés. Une référence fort typique est celle du Time Magazine, qui en fait remonter les origines à la période élisabethaine, mais pas avant : «It was Charles Darwin's eccentric mathematician cousin Francis Galton who in 1874 ignited the nature-nurture controversy in its present form and coined the very phrase (borrowing the alliteration from Shakespeare, who had lifted it from an Elizabethan schoolmaster named Richard Mulcaster)» (Matt Ridley, «What Makes You Who You Are», Time Magazine, 2 juin 2003).

${ }_{17}$ «Que il n'a chevalier en Franche / Tant valle d'escu ne de lance» (v. 5209-5210). 
aussi explicitement. L'on remarquera ce plaisir dans le ton triomphant de certaines études, qui ont représenté le roman comme un texte sinon féministe en soi, au moins proto-féministe. Les analyses de Kathleen Brahney, d'Anita Benaim Lasry et de moi-même, par exemple, ont conclu - trop vite peut-être - que l'auteur avait presque certainement voulu souligner ce dont les femmes seraient capables si elles avaient une plus grande liberté de mouvement et de rôle(s), ayant soutenu que la «culture » plutôt que la nature pourrait déterminer leur facultés et leur destin ${ }^{18}$. Je me propose, dans ce qui suit, de passer en revue les avantages ainsi que les défauts d'une telle conclusion.

\section{Études médiévales féministes et "gender studies »}

L'entrée du point de vue féministe dans les études médiévales ne fut ni facile, ni évidente ; peut-être même plus que dans les autres disciplines des humanités, les études médiévales étaient relativement plus résistantes à une approche si profondément ancrée dans les débats et les préoccupations contemporains. Au congrès international d'études médiévales de Kalamazoo, dans le Michigan, en mai 1984, E. Jane Burns, Roberta A. Krueger et Elizabeth Robertson se réunirent pour initier une collaboration qui allait produire en 1986 la Medieval Feminist Newsletter; Thelma Fenster se joindra au groupe peu après. Le but d'une telle collaboration était de redresser le manque de sessions d'orientation féministe au congrès même, ainsi que de stimuler la communication et la collaboration parmi les chercheurs ayant de tels intérêts. La distribution de la Medieval Feminist Newsletter passa de quelques douzaines d'exemplaires en 1986 à plusieurs centaines au début des années 1990 et à plus de mille en 2004 ; la publication se développa à partir de quelques pages d'annonces en un périodique à part entière. Krueger et Burns, deux des quatre fondatrices de la Newsletter, ont travaillé sur le Roman de Silence ${ }^{19}$. La Society for

\footnotetext{
${ }^{18}$ Kathleen Brahney, «When Silence Was Golden: Female Personae in the Roman de Silence», dans The Spirit of the Court: Selected Proceedings of the Fourth Congress of the International Courtly Literature Society, éd. Glyn S. Burgess and Robert A. Taylor (Cambridge : D. S. Brewer, 1985), p. 52-61; Anita Benaim Lasry, «The Ideal Heroine in Medieval Romances: Quest for a Paradigm», Kentucky Romance Quarterly 32 (1985), p. 227-43; Regina Psaki, «Introduction» au Roman de Silence (1991).

En effet Brahney suggère la possibilité qu'une femme en soit l'auteur, une idée qui fait surface de temps à autres, mais de façon atténuée, dans certaines analyses féministes. Voir Suzanna Conklin Akbari, "Nature's Forge Recast in the Roman de Silence ", dans Literary Aspects of Courtly Culture : Selected Papers from the 7th Triennial Congress of the International Courtly Literature Society, éd. Donald Maddox et Sara Sturm-Maddox (Rochester, NY : D.S. Brewer, 1994), p. 39-46 (p. 46) ; Lorraine K. Stock, «The Importance of Being Gender 'Stable' : Masculinity and Feminine Empowerment in Le Roman de Silence », Arthuriana 7.2 (1997), p. 7-34 (p. 28 et 34, note 54); Sarah Roche-Mahdi, «Introduction » à Silence : A Thirteenth-Century French Romance: «Is the author, like the heroine, a transvestite she ? Or does he just want to make us think so ?» (p. xi, n. 2) ; et Barbara Newman, «Did Goddesses Empower Women? The Case of Dame Nature», dans Gendering the Master Narrative: Women and Power in the Middle Ages, éd. Mary C. Erler et Maryanne Kowaleski (Ithaca et Londres : Cornell University Press, 2003), p. 141, ainsi que God and the Goddesses: Vision, Poetry, and Belief in the Middle Ages (Philadelphia : University of Pennsylvania Press, 2003), p. 123, parmi d'autres.
} 
datrices de la Newsletter, ont travaillé sur le Roman de Silence ${ }^{19}$. La Society for Medieval Feminist Scholarship, l'organisation scientifique qui soutient la publication (aujourd'hui le Medieval Feminist Forum), a stimulé la recherche sur le roman à travers le soutien de sessions à l'International Medieval Congress à Kalamazoo, à la Modern Language Association et dans bien d'autres congrès aux États-Unis ${ }^{20}$. L'organisation sponsorise une liste de discussion électronique, medfem-1, qui à son tour a maintenu la conversation autour du roman et qui, de temps à autres, se relance dans des échanges lorsque de nouveaux travaux paraissent, ou lorsque de jeunes collègues ou étudiants découvrent l'œuvre ${ }^{21}$.

L'essor du féminisme dans le monde académique anglophone offrit un public naturel au «valés qui est mescine» (v. 3871; encore v. 2209, 2480, 3704, 3763, 3785), dès l'apparition du roman dans la conscience collective. Barbara Newman observa que » around 1975, when the numbers of feminists studying medieval literature could be counted on the fingers of both hands, textbook accounts routinely linked the vogue for idealized female figures, such as romance heroines and allegorical goddesses, with a supposed rise in the status of women. By 1990, feminists were legion, and this consensus had largely reversed itself $»^{22}$. Ayant identifié et dirigé ses projecteurs sur un mécanisme de trame aussi attirant - une fille éduquée comme un garçon, qui devient un superbe chevalier-, la critique féministe s'est ensuite rapprochée du texte pour examiner la position de l'auteur concernant d'une telle hypothèse. D'une part, bien entendu, le roman ne peut être mis en marche que si l'on soutient que Silence puisse en effet subir complètement l'assimilation culturelle aux activités masculines et même à une identité masculine («Donques sui jo Scilentius, cho m'est avis, u jo sui nus », v. 2537-2538). Par contre, le roman se conclut (voire, à nouveau, sans se résoudre) par le dévoilement magique et le mariage conventionnel; aux yeux de nombreux lecteurs, il semble que le status quo ante est réinstallé23. Mon esquisse de ces impulsions opposées du roman au niveau de la trame est, je l'admets, assez générale, mais elle servira à la présentation des positions extrêmes de l'interprétation ${ }^{24}$.

Comme je l'ai remarqué, les paramètres de la trame pourraient sembler suggérer au premier abord, sinon une position d'auteur féministe, du moins une position en faveur de la femme, malgré les expressions explicites, de la part des personnages et du narrateur, d'opinions caricaturalement misogynes. Aux appréciations généralement positives de Brahney et de Lasry, j'ajouterais la mienne, ainsi que les travaux

${ }^{19}$ E. Jane Burns, Bodytalk: When Women Speak in Old French Literature, Philadelphia : University of Pennsylvania Press, 1993 ; Roberta L. Krueger, Women Readers and the Ideology of Genre in the Old French Verse Romance, Cambridge : Cambridge University Press, 1993.

${ }^{20}$ Le Medieval Feminist Forum est à présent géré par la Minot State University, North Dakota (http://www.minotstateu.edu/mff/).

${ }^{21}$ Pour s'abonner à medfem-l $:$ http://mailman1.u.washington.edu/mailman/listinfo/medfem-l.

${ }^{22}$ «Did Goddesses Empower Women? », p. 135.

${ }^{23}$ Voir les exceptions ci-dessous. Pour des lectures plus nuancées de la fin du roman, et de ses contextualisations, voir la note 32 .

${ }^{24}$ Je ne désire certes pas exagérer ou faire une caricature des points de vue empruntés par les nombreux critiques de Silence qui souvent se rapprochent tellement les uns des autres qu'ils exagèrent leurs propres divergences pour se différencier. 
de Lorraine K. Stock et d'Erin Labbie ${ }^{25}$. Ma propre interprétation de l'histoire comme étant assurément «non-misogyne» (1997, p. 84) est basée bien moins sur un optimisme pur et simple, que sur la conviction intime que la voix narrative est présentée tel un personnage auto-ironique, dont l'élocution cordiale mais pesante à propos de son texte, ses personnages et son sujet, est sensiblement moins sophistiquée que l'histoire qu'il raconte ${ }^{26}$. Je soutiens que l'auteur anonyme s'est inventé un substitut dans le personnage de Heldris de Cornuälle et que le public n'est pas plus invité à adopter son point de vue qu'il ne soit à s'identifier avec Eufeme, Ebain ou Merlin.

Cependant, tout au long de ces mêmes années, le roman provoquera également des interprétations absolument opposées à celle-ci. D'excellents articles et chapitres ont soutenu que le privilège accordé dans le roman à la culture est complètement illusoire, que ce n'est au fond qu'une assertion orthodoxe, même profondément misogyne, à propos de la nature et du rôle de la femme dans la société. Simon Gaunt, le premier et en même temps le plus persuasif des défenseurs du conservatisme radical du roman, soutient que l'œuvre «explores the possibility that gender is a cultural construct before firmly reinstating the status quo, in other words it offers 'proof' that the role of women is determined by biology, not upbringing ${ }^{27} »$. Cependant, en même temps, le texte n'exprime pas vraiment une conviction confortable, mais plutôt une incertitude inquiète: «Heldris's text expresses fear of women disguised as misogyny, and that, ironically, this misogyny draws attention to the very anxiety he wishes to conceal». D'après Gaunt, les dires du narrateur représentent en effet les convictions de l'auteur: il n'attribue, dans son analyse, aucun sens ou aucune raison à une divergence consciente entre l'auteur et le narrateur. Cependant, cette séparation, que je présente comme étant intentionnelle - un concept non sans problèmes, j'en suis consciente - est interprétée par Gaunt comme un reflet, à l'intérieur du texte, de l'inconscient politique: «Perhaps, despite the repressive dénouement, $[\ldots]$ one can hear distorted echoes of the voices of silenced and marginalized women », puisque le texte même exprime «precisely what the poet ostensibly seeks to repress ». E. Jane Burns et Roberta A. Krueger s'accordent sur le fait que l'insistance même du roman, à propos de la nature inférieure de la femme, ouvre un nouvel espace discursif aux lecteurs, de sorte qu'ils puissent contester une telle assomption. Elles se rangent ainsi dans une certaine veine d'études médiévales féministes qui voit des «oppressive structures, however monolithic they may seem or claim to be, as necessarily fragile, permeable, and open to resistance $»^{28}$. En d'autres

\footnotetext{
${ }^{25}$ Psaki 1991, «Introduction», et «The Modern Editor and Medieval 'Misogyny' : Text Editing and Le Roman de Silence», Arthuriana 7.2 (1997), p. 78-86; pour Stock, voir note 18. Erin Labbie en particulier (voir n. 10) lit le Roman de Silence comme une critique intransigeante de la subordination des femmes dans une société patriarcale.

${ }^{26}$ L'hypothèse du narrateur auquel il ne faut pas se fier (le «unreliable narrator» de Wayne Booth) fut acceptée par Suzanne Kocher, «Narrative Structure of the Roman de Silence: Lessons in Interpretation», Romance Notes 42 (2002), p. 349-358 (ici p. 349); de même Barbara Newman (2003, p. 132) et Haidu (2004, p. 252).

${ }^{27}$ Simon Gaunt, «The Significance of Silence », Paragraph, 13 (1990), p. 202-216 ; toutes les citations de ce paragraphe apparaissent à la p. 203.

${ }^{28}$ E. Jane Burns, Courtly Love Undressed, p. 69.
} 
termes, ces trois critiques cherchent donc à mitiger leurs conclusions à propos de la charge misogyne du texte en accentuant le potentiel contestataire de son intransigeance. D'autres lecteurs interprètent la prise de position de l'auteur strictement comme catégoriquement conservatrice en s'appuyant sur le fait que Heldris présente la re-féminisation et le mariage de Silence comme un «happy ending » et le redressement de l'ordre social ${ }^{29}$; ou comme l'a écrit Simon Gaunt: "Heldris portrays the return to the order he wishes to endorse as the triumph of nature over nurture ».

Sharon Kinoshita exprime ainsi cette division dans la critique: «The narrative that unfolds invites two contrary conclusions. The first casts Silence as an oppositional text, focusing on how it renegotiates boundaries between sex and gender. The second, in contrast, emphasizes the way the text recontains the subversion it unleashes $»^{30}$. Un alignement de toute la critique publiée du Roman de Silence selon un tel spectre pourrait être intéressant, mais en fin de compte cela n'est ni utile, ni nécessaire. Aucun des critiques ayant travaillé sur la «misogynie» ou la «philogynie» du roman, ou sur son statut d'œuvre d'art conservatrice ou contestataire, n'ignore l'évidence qui peut défier sa prise de position ; chacun soupèse des impulsions, des passages et des accents différents dans son identification des centres de valeurs positives du texte. Il est certain qu'aucun critique travaillant sur ce texte ne se doit de choisir l'un des deux camps : Peggy McCracken, Lynne Dahmen et Barbara Newman ont ainsi présenté des opinions refusant de telles prises de position monolithiques concernant Heldris ou son texte, et Elizabeth Waters conclut que le texte incorpore «both complicity in and subversion of the status quo », soit que les deux interprétations contradictoires peuvent être soutenues simultanément ${ }^{31}$. Peter Haidu confirme qu'il ne faut pas réduire le texte à l'une ou l'autre opinion en affirmant que telle n'est, en général, jamais la responsabilité d'un texte médiéval en langue vulgaire: "The ideology of the exemplum institutes one meaning per tale. Otherwise, medieval texts rarely try to specify a single, univocal meaning. Instead, they give local interpretations or deploy markers to signal the kinds of questions to be asked of their narratives $»^{32}$. En ce qui me concerne, Haidu a entièrement raison quand il insiste sur le fait que le Roman de Silence nous offre des indices pour nous aider dans notre quête à l'intérieur du texte et il a raison de proclamer un tel modus operandi pour les textes médiévaux en général, plutôt que de voir ce roman comme unique en son ambiguïté : «Above all, the medieval vernacular text is a site which attracts and deploys contradictory systems of value and keeps those polysemous, contradictory values in play for the reader to meditate. This text does not boast of

\footnotetext{
${ }^{29}$ Kathleen Blumreich, "Lesbian Desire in the Old French Roman de Silence », Arthuriana 7.2 (1997), p. 47-62 ; Caroline A. Jewers, «The Non-Existent Knight: Adventure in Le Roman de Silence », Arthuriana 7.2 (1997), p. 87-110.

${ }^{30}$ Sharon Kinoshita, «Heldris de Cornuälle's Roman de Silence and the Feudal Politics of Lineage », PMLA 110.3 (1995), p. 397-409 (ici p. 402).

${ }^{31}$ Peggy McCracken, "'The Boy Who Was a Girl' : Reading Gender in the Roman de Silence », Romanic Review 85 (1994), p. 515-534 ; Lynne Dahmen, « Sacred Romance : Silence and the Hagiographical Tradition », Arthuriana 12.1 (2002), p. 113-122 ; Newman, op. cit. ; Elizabeth Waters, "The Third Path: Alternative Sex, Alternative Gender in Le Roman de Silence », Arthuriana 7.2 (1997), p. 35-46, ici p. 37.

${ }^{32}$ Peter Haidu, op cit., p. 242.
} 
the classical unities, or the fictive semantic unity of the theoretical exemplum. Contradiction is the form of logical coherence that defines a semiotic text $»^{33}$.

Enfin, je suis convaincue que la question de savoir où, dans ce spectre spécifique, il nous faut situer le roman, est à présent dépassée par la prise de conscience que le roman présente et problématise bien d'autres spectres. Tout particulièrement, la «queer theory » a repositionné le débat de la politique sexuelle du Roman de Silence, un débat qui avait exposé - de façon peu critique, il est vrai- le couple «homme hétérosexuel / femme hétérosexuelle» comme l'opposition principale du texte $^{34}$. Mais, au-delà des «gender studies » en général, gisent des mondes nouveaux de critique de Silence, que l'on commence à découvrir.

\section{Le Roman de Silence au-delà des «gender studies»}

Dans mon introduction du premier numéro spécial d'Arthuriana sur le Roman de Silence, j'écris : «At some point this romance may lose its specificity as a focus for gender criticism, and be mined instead for information on child-rearing in the Middle Ages, on the practice of itinerant minstrelsy, or on the many legal issues it explores in such careful detail $»^{35}$. Ce jour était en effet déjà arrivé, comme en témoignera la suite de cet essai. Quant à ces trois exemples, que j'avais choisis un peu au hasard, Nicole Clifton était sur le point de publier un article sur Silence et sur d'autres personnages dans le contexte de traités latins de pédagogie ${ }^{36}$. John Fleagle et Shira Kammen, deux musiciens renommés et experts en musique ancienne, avaient déjà créé et exécuté une version du Roman de Silence qui s'intéressa à la trame secondaire des ménestrels, dont je parlerai plus loin. Kathleen Blumreich travaillait sur une analyse, présentée au congrès triennal de l'International Courtly Literature Society en 1998, des affaires légales mises à l'avant-plan dans le roman.

Mais, comme je l'ai indiqué ci-dessus, de telles approches, non pas particulièrement orientées vers les «gender studies", avaient déjà fait surface dès l'avènement de la critique sur Silence. L'attention de R. Howard Bloch aux impératifs politiques et généalogiques a été reprise par Sharon Kinoshita, dans un article d'une grande force et influence et qui cherchait à « move from a poetics to a politics of Silence $»^{37}$. Dans un second article, elle soutient que le roman en général, et celuici en particulier, sert à mettre en scène un débat urgent : «the monarchical exploitation of the feudal politics of lineage $»^{38}$. La trame entière, après s'être révélée si attirante pour la critique féministe, sert principalement à lancer la recherche dans une

\footnotetext{
${ }^{33}$ Peter Haidu, op. cit., p. 261.

${ }^{34}$ Voir Kathleen Blumreich, art. cit. ; Robert L.A. Clark, «Queering Gender and Naturalizing Class in the Roman de Silence », Arthuriana 12.1 (2002), p. 50-63 ; Elizabeth Waters, art. cit. ; Valerie Hotchkiss, Clothes Make the Man: Female Cross-Dressing in Medieval Europe (New York: Garland, 1996), p.105-129.

35 «Introduction», Arthuriana 7.2 (1997), p. 6.

${ }^{36}$ «The Point of Education: Views from Medieval France and England», Children's Literature Association Quarterly 23 (1998), p. 12-21.

${ }^{37}$ Sharon Kinoshita, art. cit. (1995), p. 398.

${ }^{38}$ Sharon Kinoshita, «Male-Order Brides : Marriage, Patriarchy, and Monarchy in the Roman de Silence », Arthuriana 12.1 (2002), p. 64-75 ; ici p. 64.
} 
tout autre direction ${ }^{39}:$ «For under the cover of Silence's cross-dressing and refeminization, the romance reformulates the way bodies matter in the thirteenth-century imaginary, redefining the function of the medieval nobility as not military service but genealogical reproduction $»^{40}$. Comme Kinoshita l'a indiqué, il est ardu d'installer le roman dans son contexte spécifique, local et historique, avec beaucoup de précision, à cause du manque d'informations concrètes à propos du texte et de sa genèse ${ }^{41}$. Toutefois, des recherches à fondement historique sur le XIII ${ }^{\mathrm{e}}$ siècle, sur le nord de la France et les Pays-Bas, sur les relations entre (ce que sont aujourd'hui) la France et l'Angleterre, sont apparues dans des communications lors de congrès et dans des articles publiés ${ }^{42}$.

En 1998, un symposium intitulé «The Maiden-Knight: The Roman de Silence in the Romance Tradition» fut organisé à l'Université de l'Oregon. Ce titre suggère le désir d'atténuer la rhétorique du caractère unique du texte et de le réinsérer dans ses contextes, qu'ils soient littéraire, historique ou culturel. Le symposium engendra la publication d'un second numéro spécial d'Arthuriana sur le Roman de Silence (2002), ayant pour but de rassembler un groupe de travaux plus hétérogènes que dans le premier. La collection présente des sujets comme la crise des catégories ; le rang et l'idéologie de la hiérarchie sociale ; la prose médiévale japonaise ; les lois féodales de l'héritage; les hagiographies; les enluminures des manuscrits de romans; la tradition de l'homme sauvage ; L'Estoire Merlin; la tradition des ménestrels; le discours lyrique; la pédagogie médiévale; et enfin, mais d'une grande importance, des stratégies didactiques pour l'enseignement du roman ${ }^{43}$. Une table ronde intitulée «Le Roman de Silence: Beyond Gender Studies» fut organisée en 2000 à l'International Medieval Congress. Je tiens à le répéter, les gender studies ne furent nullement écartées, car il serait difficile de travailler de façon constructive sur le roman sans faire référence à cette dimension de la trame ; les chercheurs ont simplement absorbé et assimilé les «gender studies » dans une variété d'autres perspectives sur le roman. Karen Pratt, par exemple, examine l'«intertextual and intergeneric comedy» du roman, ainsi que son «verbal, structural, and situational humour », avec une attention particulière aux commentaires et aux plaisanteries misogynes ${ }^{44}$.

${ }^{39}$ Robert Clark, art. cit, et Robert Sturges, «The Cross-Dresser and the Juventus : Category Crisis in Silence », Arthuriana 12.1 (2002), p. 37-49, s'occupent également de la façon dont le roman engage des questions de rang, que nous appellerions, aujourd'hui, de classe.

${ }^{40}$ Sharon Kinoshita, art. cit. (1995), p. 398.

${ }^{41}$ Sharon Kinoshita, art. cit. (2002), p. 64.

${ }^{42}$ H.J. Tanner, communication; Katie Keene, «Cherchez Eufeme: The Evil Queen in Le Roman de Silence », Arthuriana 14.3 (2004), 3-22 ; Craig A. Berry, «What Silence Desires : Female Inheritance and the Romance of Property », dans C.A. Berry et H. Hayton éd., Translating Desire in Medieval and Early Modern Literature (Tempe, Az: Arizona Center for Medieval and Renaissance Studies, 2005), p. 191-206. Pour le contexte littéraire, voir Haidu et Jewers.

${ }^{43}$ Il faut noter ici que Bonnie Wheeler, éditeur d'Arthuriana, est à l'origine de 21 des 47 articles sur le Roman de Silence publiés dans des revues jusqu'à ce jour en langue anglaise.

${ }^{44}$ Karen Pratt, «Humour in the Roman de Silence», Arthurian Literature XIX: Comedy in Arthurian Literature, éd. Keith Busby et R. Dalrymple (Cambridge: D.S. Brewer, 2003), p. 87-103 (ici p. 87). 
Enfin, le manuscrit même, le Mi LM 6 de la bibliothèque Hayward de l'Université de Nottingham, est à présent l'objet d'une étude bien méritée, et non plus uniquement comme véhicule pour l'un ou l'autre des textes qu'il transmet ${ }^{45}$. Keith Busby a produit, jusqu'à ce jour, l'analyse la plus approfondie du manuscrit en langue anglaise et il remarque, non sans une certaine âpreté, que la critique, orientée le plus souvent vers les «gender studies", a délaissé le manuscrit même en tant que ressource pour l'interprétation du roman. Son livre en deux volumes Codex and Context situe le manuscrit Mi LM 6 dans le spectre complet des manuscrits contenant des textes narratifs en vers en ancien français ${ }^{46}$.

\section{Reprises et exécutions : adaptations musicales et théâtrales en langue an-} glaise

La trame du Roman de Silence semble avoir attiré l'attention d'au moins deux romanciers francophones. Le Chevalier Silence de Jacques Roubaud et L'Enfant de sable de Tahar Ben Jelloun accusent des liens très forts, sinon de véritables dettes envers le texte médiéval ${ }^{47}$. Dans la production culturelle anglophone, le thème de la performance dans le roman, ainsi que sa disponibilité pour des cours universitaires dans la traduction de Roche-Mahdi, a commencé à produire plusieurs versions pour la scène. Elles varient du concert de musique ancienne aux compositions nouvelles, jusqu'aux versions théâtrales, proposées par des conteurs, ou des exécutions entièrement dramatiques du roman. Chacune de ces nouvelles incarnations du Roman de Silence accentue une dimension différente du texte, qui nécessairement ajoute une dimension neuve à ma compréhension de l'œuvre.

John Fleagle et Shira Kammen ont présenté en 1996, au fameux Festival des Flandres à Bruges, Le Roman de Silence sous forme d'hybride lyrico-narratif. Leur programme combinait des passages déclamés du roman avec des pièces vocales et instrumentales - médiévales ou composées dans un style de l'époque par les deux musiciens -, glosant ainsi l'histoire pour en propulser le développement. On ne s'étonnera pas du fait que cette version mit à l'avant-plan l'épisode des jongleurs, tout en s'imaginant de quelle façon l'histoire aurait pu avoir été communiquée au public médiéval, qui n'aurait certainement pas lu, en silence et en toute solitude dans un livre, le texte de Heldris. Cette version était absolument envoûtante et les musiciens se servirent entièrement de la force de la mélodie et de l'exécution vocale pour faire sentir au public le pathos de certains passages, qui dans le roman ne sont franchement pas des plus touchants - des passages qui se limitent à décrire une émotion,

${ }^{45}$ Voir P. Allen, art. cit. ; F. Cowper, art. cit. ; Michelle Bolduc, «Images of Romance: The Miniatures of Erec et Enide and Le Roman de Silence », Arthuriana 12.1 (2002), p. 101-112; et récemment Danièle James-Raoul, «La poétique de la lettrine dans Le Roman de Silence », Cahiers de Recherches Médiévales 12 (2005), p. 227-245.

${ }^{46}$ Codex and Context: Reading Old French Verse Narrative in Manuscript (Amsterdam: Rodopi, 2002).

${ }^{47}$ Norris Lacy, «Echoes of Silence : From Heldris de Cornuälles to Jacques Roubaud », dans Chançon legiere a chanter, éd. Karen Fresco et Wendy Pfeffer (Birmingham, AL : Summa, à paraître). Outre Erika E. Hess (voir note 12), voir aussi Florence Ramond Jurney, «Secret Identities: (Un)Masking Gender in Le Roman de Silence by Heldris de Cornouaille and L'enfant de sable by Tahar Ben Jelloun », Dalhousie French Studies 55 (2001), p. 3-10. 
plutôt que de la transmettre au public. Par exemple, le passage dans lequel Eufemie se plaint de la douleur que lui cause l'amour (v. 777-797) fut transformé, d'une simple peine d'amour classique, en une émotion d'une intensité élevée, grâce à la mélodie ajoutée, empruntée au Lai 6 du MS 2542 de Vienne. La description raffinée de l'offrande de la beauté parfaite à l'enfant Silence de la part de Nature ne fut pas seulement fascinante, mais touchante, lorsqu'elle fut enrichie de «A globo veteri » (Florence, Biblioteca Laurenziana, Plut. 29.I) de Pierre de Blois.

Fleagle et Kammen ont présenté leur Roman de Silence en deux versions l'une en ancien français et en latin, l'autre en anglais et en latin - à l'occasion de nombreux concerts de musique médiévale dans des universités, festivals et séries de concerts en Amérique du Nord, contribuant ainsi à divulguer l'histoire en dehors des milieux purement académiques. Depuis le décès, en 1999, de John Fleagle, il ne nous reste aucun enregistrement complet (audio ou vidéo) du spectacle, mais de nouvelles initiatives musicales sont en cours et en contemplation auprès de spécialistes de musique médiévale, notamment le soprano Anne Azéma, qui, comme John Fleagle, travaille également avec Shira Kammen ${ }^{48}$. Linda Marie Zaerr, de la Boise State University, est en train d'écrire une adaption théâtrale du Roman de Silence, qui sera produite en automne 2006. Sa version sera en anglais avec des interpolations en ancien français, utilisant «des chanteurs et trois vièles (si tout va bien) et un cast de plusieurs dizaines de personnes $»$. Zaerr s'est promise de situer la production dans une bibliothèque universitaire : «Je ne pouvais pas résister à l'idée d'une production bruyante de Silence dans une bibliothèque ${ }^{49} \%$.

Un nouvel oratorio de Katherine Saxon, «The Return of Silence», met en musique les vers 3476-3676. Lorsque Silence revient dans la maison de ses parents, après avoir passé quatre années comme ménestrel, «il» découvre que tous les ménestrels ont été banni de Cornouaille, sous peine de mort. L'oratorio consiste en un prologue et quatre scènes (Arrival ; At Court; In the Chambers ; Home) et utilise trois solistes (Silence, soprano ; Cador, basse; et le Vieil Homme/Merlin, ténor), tandis que le chœur adopte les rôles du narrateur et du peuple. D'après Saxon, l'accompagnement instrumental trahit une forte influence de la musique modale du Moyen Âge. Cette version toute fraîche met à l'avant-plan des éléments différents du roman. À propos de sa version de l'histoire, Saxon dit:

Plutôt que de me concentrer sur les désavantages d'être femme dans un monde masculin, j'ai tenté de capter le danger d'être quelqu'un d'autre que soi-même. Silence, en brave fillette, suit si bien les instructions de ses parents qu'elle y laisse presque sa peau ; elle a travesti son propre être au-delà de toute reconnaissance et elle est sur le point d'être tuée. [...] La tragédie de Silence est qu'elle ne peut jamais être complètement elle-même, femme, ménestrel et chevalier, et son silence à la fin du roman, ainsi que sa présence silencieuse sur la scène à la fin du «retour de Silence » (lorsque tout le monde se réunit dans le chœur « My heart is full of joy ») est le symbole de sa défaite finale, malgré ses efforts énormes. ${ }^{50}$

\footnotetext{
${ }^{48}$ Voir www.anneazema.com.

${ }^{49}$ Communication personnelle du 26 mai 2006.

${ }^{50}$ Communication personnelle du 15 juin 2006.
} 
Le livret et la mise en musique de Saxon ne fonctionnent pas seulement comme une compensation pour les éléments narratifs demeurés à l'extérieur de sa composition, mais ils ajustent le point focal, de sorte que le déguisement du protagoniste, ainsi que sa signification, soient accentués - le tout fait de la façon la «plus adaptée au médium choisi ».

Dolores Hydock, conteuse professionnelle et actrice, créa une version narrative de l'histoire de Silence, qu'elle intitula "Silence: A Medieval Adventure in Story and Song », accompagnée musicalement par l'ensemble PanHarmonium. Présentée à l'International Medieval Congress de Kalamazoo en 2005, cette version est intéressante et très vivante. Hydock rejeta complètement la voix narrative un peu pompeuse et hystérique du roman, pour la remplacer par une conteuse de son invention, une vieille femme avec un foulard, une canne et un accent londonien Cockney un peu gratuit, mais amusant. En utilisant cet étrange personnage, elle obtint un bon effet en insistant sur certaines incongruités de l'histoire, que Heldris avait laissé dépourvues de commentaires. Par exemple, au début du roman, le roi Ebain - que le narrateur avait par trop loué - sera réduit à trembler comme une feuille devant l'horrible dragon: «Li rois Ebayns fort s'esmarist» (v. 356) ; «Or a li rois Ebains grant doute» (v. 364). En racontant ce passage de l'histoire, Hydock s'adressa au public en disant, non sans sous-entendus satiriques: "Well, I said he was wise, I never said he was brave». Heldris n'a fait aucune allusion à l'incongruité de ce roi admirable, qui meurt de peur, mais Hydock ne fit qu'indiquer les incohérences dans la caractérisation du roi, mettant ainsi en valeur sa propre révision de la voix narrative $^{51}$. Il existe un enregistrement audio de cette version, qui amusera tant les jeunes que le public adulte ${ }^{52}$.

L'histoire de Silence se prête également à une adaptation théatrale. Une initiative fascinante est celle des professeurs Evelyn Birge Vitz, Nancy Freeman Regalado et Marilyn Lawrence, qui enseignent le roman médiéval à travers la performance théâtrale. Elles invitent leurs étudiants à jouer des scènes clés de romans, et de nombreux exemples tirés du Roman de Silence apparaissent sur le site internet du projet ${ }^{53}$. Moira Buffini a écrit, en 1998, une pièce (primée par la critique) intitulée Silence, qui fut montée aux États-Unis et en Grande-Bretagne. Située à la fin du $\mathrm{XI}^{\mathrm{e}}$ siècle, la pièce ne reproduit absolument pas la trame du Roman de Silence, mais la présence d'un personnage nommé 'Lord Silence of Cumbria', une fille déguisée en garçon, trahit une certaine influence du roman. Il ne reste plus qu'à un scénariste entreprenant d'en faire un scénario : bien que je craigne une version de music-hall à la Broadway, j'ai de bons espoirs pour un film acceptable.

\section{Conclusion}

Tout ceci nous ramène à notre point de départ : le Roman de Silence est, avec sa trame de déguisement et son interrogation ouverte des relations entre le sexe biologique et la gender identity, une histoire qui fonctionne parfaitement à notre

\footnotetext{
${ }^{51}$ Lorraine Stock observa la même caractérisation indirecte, art. cit., p. 13.

${ }^{52}$ Pour des informations à propos des programmes et enregistrements de Hydock, on peut contacterdolores@storypower.org ou consulter www.storypower.org.

53 «Performing Medieval Narrative Today: A Video Showcase», sur le site euterpe.bobst.nyu.edu/mednar/
} 
époque. Une kyrielle de romans et de films lui empruntent un ensemble de thèmes similaires et nous pouvons nous attendre à d'autres parallèles ${ }^{54}$. L'attirance initiale vers le Roman de Silence fut, pour les chercheurs anglophones, le rapport entre le sexe et le gender, la nature et la culture, le langage et l'interprétation. Barbara Newman l'a ainsi synthétisé : les «antinomies [...] within Nature's compass - heterosexual versus homosexual desire, biological versus social gender, reproduction versus abstinence, plain speech versus linguistic play, female achievement versus unjust laws - converge almost uncannily in Silence $»^{55}$. Ces oppositions donnent ensuite lieu à une série de problèmes qui s'entrelacent, comme le rôle des femmes dans la société féodale et dans la production littéraire courtoise ; l'influence de la misogynie cléricale dans la culture courtoise en général; les intersections possibles de structures sociales et idéologiques anti-féministes d'une part, et de l'autre, les réponses féministes ou proto-féministes à ces structures, ainsi que leur contestation de celles-ci; la nature des rôles des sexes et leur insertion dans la société et l'idéologie ; l'exploration du gender comme labile, instable et ancré dans la performance plutôt que dans la biologie ; la nature de la gender identity dans un monde de rôles fortement dictés et hiérarchisés ; et la nature de l'identité en général, perçue et sentie, jouée et essentielle, publique et privée.

L'on reconnaîtra facilement ces intérêts comme typiques du dernier tiers du $\mathrm{XX}^{\mathrm{e}}$ siècle et une telle spécificité a engendré le doute qu'ils puissent illuminer de façon transparente la production culturelle d'une époque bien plus ancienne, une époque qui est, en fait, souvent si différente de la nôtre. Ce problème épistémologique et méthodologique fut l'objet de débats ardents dans le monde universitaire et n'a certes pas encore été résolu. Le degré par lequel le Moyen Âge - tant la réalité historique que l'imaginaire - peut être «transparent » pour nos siècles est fort discutable, bien que chaque participant à ce débat considère sa propre approche comme la plus prometteuse en vue de négocier la spécificité du passé. Il est facile de lancer l'accusation d'anachronisme et en ce qui me concerne, je voudrais ici seulement insister sur le fait que le Roman de Silence engendre explicitement (mais sans les résoudre), dans sa trame, dans sa caractérisation et dans ses interpolations narratives, des débats se rapportant au gender, à l'identité, à la nature et à la culture, qui l'ont rendu appétissant aux chercheurs et aux artistes nord-américains d'aujourd'hui, dans un monde culturel trop occupé à reprendre les mêmes discussions dans la bataille pleine de rancœur que nous appelons, aux États-Unis, les «guerres culturelles».

F. Regina Psaki

University of Oregon

\footnotetext{
${ }^{54}$ Exemples de travaux non dérivés du roman, mais proches de lui sont entre autres : Louise Erdrich, The Last Report on the Miracles at Little No-Horse ; Jeffrey Eugenides, Middlesex; Boys Don't Cry; Shakespeare in Love; et Mulan.

${ }^{55}$ God and the Goddesses (2003), p. 122-123.
} 


\section{ANNEXE \\ Bibliographie complémentaire sur Le Roman de Silence}

\section{MANUSCRIT ET ÉTUDES CODICOLOGIOUES}

Le Roman de Silence: MS Mi LM 6, University Library, Nottingham, ff. 188r-223v. BUSBY Keith, Codex and Context: Reading Old French Verse Narrative in Manuscript, Amsterdam: Rodopi, 2002. The main discussion of Mi. LM. 6 is on p. 415-421; other refs. on p. 364 (n. 215), 413, 427, 481, 515-516, 690 (n. 201), 692-693.

COWPER Frederick A. G., «Origins and Peregrinations of the Laval-Middleton Manuscript », Nottingham Medieval Studies 3 (1959), p. 3-18.

COWPER Frederick A. G., "The New Manuscript of Ille et Galeron", Modern Philology, 18 (1920-1921), p. 601-608.

STEVENSON W. H., Report on the Manuscripts of Lord Middleton at Wollaton Hall, Nottinghamshire, Historical Manuscripts Commission, 1911. Sur le Mi LM 6, voir les p. 221-234 ; sur le Roman de Silence les p. 224-225.

\section{TRADUCTION}

LASRY, Anita Benaim, El Libro de Silence, Madrid : Ediciones Siruela (Selección de Lecturas Medievales, $\left.{ }^{\circ} 17\right), 1986$.

III. ÉTUDES

BERRY, Craig A., «What Silence Desires : Female Inheritance and the Romance of Property », Translating Desire in Medieval and Early Modern Literature, éd. Craig A. Berry et Heather Hayton, Tempe, AZ: Arizona Center for Medieval and Renaissance Studies, 2005, p. 191-206.

BLOCH, R. Howard, Etymologies and Genealogies : A Literary Anthropology of the French Middle Ages, Chicago : U Chicago P, 1983, p. 195-197, 205.

BOUCHET Florence, «Le Silence de la travestie : Le Roman de Silence (XIII ${ }^{\mathrm{e}}$ siècle) », Clio : Histoire, Femmes et Sociétés 10 (1999), p. 137-144.

BULLOUGH Vern L. et Bonnie, «Cross-Dressing and Social Status in the Middle Ages », Cross-Dressing, Sex and Gender, Philadelphia: University of Pennsylvania Press, 1993, p. 45-73, et surtout p. 57-60.

BULLOUGH Vern L., "On Being a Male in the Middle Ages», éd. Clare A. Lees, Thelma Fenster et Jo Ann McNamara, Medieval Masculinities : Regarding Men in the Middle Ages, Minneapolis: University of Minnesota Press, 1994, p. 31-45.

BULLOUGH Vern L., «Sex in History : A Redux », éd. Jacqueline Murray et Konrad Eisenbichler, Desire and Discipline: Sex and Sexuality in the Premodern West, Toronto, ON : University of Toronto Press, 1996, p. 3-22.

BURNS E. Jane, Bodytalk: When Women Speak in Old French Literature, Philadelphia : U Pennsylvania P, 1993, p. 243-245, 249.

BURNS E. Jane, «Courtly Love: Who Needs It? Recent Feminist Work in the Medieval French Tradition », Signs : Journal of Women in Culture and Society 27.1 (2001), p. 23-57, et surtout p. 32, 43-46. 
BUSBY Keith, «'Plus acesmez qu'une popine' : Male Cross-Dressing in Medieval French Narrative », éd. Karen J. Taylor, Gender Transgressions : Crossing the Normative Boundary in Old French Literature, New York and London: Garland Publishing, 1998, p. 45-59.

CARRETO Carlos F.C., «Le Nom-palimpseste ou les contradictions du désir : Désigner dans le récit médiéval (XII ${ }^{\mathrm{e}}-\mathrm{XIII}{ }^{\mathrm{e}}$ siècles) », éd. Danielle Buschinger, Études médiévales, Centre d'Études Médiévales, Université de PicardieJules Verne, Amiens, France, 1999.

CARRETO Carlos F.C., «La Parole transpercée: Pour une esthétique du silence dans le roman arthurien", éd. Danielle Buschinger, Études médiévales, Centre d'Études Médiévales, Université de Picardie-Jules Verne, Amiens, France, 1999.

CLIFTON Nicole, "The Point of Education: Views from Medieval France and England», Children's Literature Association Quarterly 23 (1998), p. 12-21.

DIETZMAN Sara Jane, «En guize d'omme»: Female Cross-Dressing and Gender Reversal in Four Medieval French Texts, Ph.D., University of Virginia, 2005.

ENDERS Jody, «The Farce and Folly of Female Authority in a Sixteenth-Century View of the Roman de Silence», Romance Notes 33 (1993), p. 33-37.

FERRANTE Joan, «Public Postures and Private Maneuvers : Roles Medieval Women Play »,Women and Power in the Middle Ages, éd. Mary Erler et Maryanne Kowaleski, Athens, GA : University of Georgia Press, 1988, p. 213229.

GIANNACHI G., «Parole e silenzi da le Roman de silence», éd. C. Churchill, Divina, arte femminile in scena, Turin : Tirrenia Stampatori, 1996, p. 101-114.

HAIDU Peter, «Problematizing Identity : Silence», chapitre 11 de The Subject Medieval / Modern: Text and Governance in the Middle Ages, Stanford, CA : Stanford University Press, 2004, p. 239-265.

HESS Erika E., «Passing the Test of Truth : Gender as Performance in Two French Narratives, Medieval and Modern», Cincinnati Romance Review 17 (1998), p. 42-48.

HESS Erika E., Cross-Dressers, Werewolves, Serpent-Women, and Wild Men : Physical and Narrative Indeterminacy in French Narrative, Medieval and Modern, Diss. U of Oregon, 2000.

HESS Erika E., Literary Hybrids : Cross-Dressing, Shapeshifting, and Indeterminacy in Medieval and Modern French Narrative, New York and London: Routledge, 2004.

HOTCHKISS Valerie R., Clothes Make the Man: Female Cross-Dressing in Medieval Europe, New York: Garland, 1996, p. 105-129.

JURNEY Florence Ramond, «Secret Identities : (Un)Masking Gender in Le Roman de Silence by Heldris de Cornouaille and L'Enfant de sable by Tahar Ben Jelloun », Dalhousie French Studies 55 (2001), p. 3-10.

KRUEGER Roberta L., «Beyond Debate: Gender in Play in Old French Courtly Fiction », éd. Fenster, Thelma S. et Clare A. Lees, Gender in Debate from the Early Middle Ages to the Renaissance, New York: Palgrave, 2002, p. 79-95, et surtout p. 87-91. 
LACY Norris L., «Echoes of Silence : From Heldris de Cornuälles to Jacques Roubaud», Chançon legiere a chanter, éd. Karen Fresco et Wendy Pfeffer, Birmingham, AL : Summa, à paraître.

NEWMAN Barbara, "Testing the Norms : Nature, Nurture, Silence », chapitre 3 de God and the Goddesses: Vision, Poetry, and Belief in the Middle Ages, Philadelphia : University of Pennsylvania Press, 2003, p. 122-134.

NEWMAN Barbara, «Did Goddesses Empower Women? The Case of Dame Nature», éd. Mary C. Erler et Maryanne Kowaleski, Gendering the Master Narrative: Women and Power in the Middle Ages, Ithaca and London: Cornell University Press, 2003, p. 135-155.

SZKILNIK Michelle, «The Grammar of the Sexes in Medieval French Romance», éd. Karen J. Taylor, Gender Transgressions: Crossing the Normative Boundary in Old French Literature, New York and London : Garland Publishing, 1998, p. 61-88. 